\title{
Effect of Rho GTPase activating protein 9 combined with preoperative ratio of platelet distribution width to platelet count on prognosis of patients with serous ovarian cancer
}

\author{
Yang Shen ${ }^{1}$, Haibo $\mathrm{Xu}^{2}$, Zhihong Guan ${ }^{1}$, Mengmeng $\mathrm{Lv}^{1}$, Tianye Qian ${ }^{1}$, Yuzhong Wu ${ }^{1}$ \\ ${ }^{1}$ Department of Gynecologic Oncology, Jiangsu Cancer Hospital and Jiangsu Institute of Cancer Research and The Affiliated Cancer Hospital of \\ Nanjing Medical University, Nanjing, China; ${ }^{2}$ Department of Gynecology, Affiliated Cancer Hospital of Nantong University, Nantong, China \\ Contributions: (I) Conception and design: Y Shen; (II) Administrative support: None; (III) Provision of study materials or patients: Z Guan, T Qian; (IV) \\ Collection and assembly of data: H Xu; (V) Data analysis and interpretation: M Lv, Y Wu; (VI) Manuscript writing: All authors; (VII) Final approval \\ of manuscript: All authors. \\ Correspondence to: Yuzhong Wu. Department of Gynecologic Oncology, Jiangsu Cancer Hospital and Jiangsu Institute of Cancer Research and The \\ Affiliated Cancer Hospital of Nanjing Medical University, 42 Baiziting Street, Nanjing 210009, China. Email: wuyuzhong1127@126.com.
}

Background: This study aimed to investigate the relationship between Rho GTPase activating protein 9 (ARHGAP9) combined with preoperative ratio of platelet distribution width to platelet count (PDW/PLT) and patients prognosis with serous ovarian cancer.

Methods: The clinical data of 80 patients with serous ovarian cancer treated in Jiangsu Cancer Hospital from May 2011 to May 2016 were analyzed retrospectively. We verified ARHGAP9 expression in The Cancer Genome Atlas (TCGA) database, then detected messenger RNA (mRNA) expression encoding ARHGAP9 in ovarian cancer tissue samples using reverse transcription quantitative polymerase chain reaction (RT-qPCR). These patients were divided into an ARHGAP9 low-expression group and an ARHGAP9 high-expression group. The optimal critical value of PDW/PLT was determined by receiver operating characteristic (ROC) curve. The patients were divided into low PDW/PLT group and high PDW/PLT group. Kaplan-Meier method and log-rank test were used for univariate survival analysis, Cox regression method was used for multivariate analysis, and then a nomogram was constructed for internal verification.

Results: The ARHGAP9 protein was highly expressed both in TCGA serous ovarian cancer database and the serous ovarian cancer tumor tissues. There were significant differences in menstrual status, the International Federation of Gynecology and Obstetrics (FIGO) stage and grade between the ARHGAP9 low expression group and ARHGAP9 high expression group (all $\mathrm{P}<0.05$ ). There were significant differences in FIGO stage, lymph node metastasis, and ascites between the low PDW/PLT group and high PDW/PLT group (all $\mathrm{P}<0.05)$. Finally, 80 patients were included, with a mortality rate of $45.0 \%$ and a survival rate of $55.0 \%$; the median progression-free survival (PFS) was 19 months, and the median overall survival (OS) was 62.5 months. Cox multivariate analysis showed that PDW/PLT and ARHGAP9 were independent risk factors for tumor progression ( $\mathrm{P}=0.026$ and $\mathrm{P}=0.028$, respectively). In the internal validation, the $\mathrm{C}$-index of the nomogram was 0.6518 [ $95 \%$ confidence interval (CI): 0.5685 to 0.7352 ], and the prediction model had certain accuracy.

Conclusions: ARHGAP9 and PDW/PLT Decrease can significantly prolong OS and PFS in serous ovarian cancer patients. Therefore, ARHGAP9 can be used as a new predictive biomarker and may be related to the immune infiltration of serous ovarian cancer.

Keywords: Serous ovarian cancer; Rho GTPase activating protein 9 (ARHGAP9); platelet distribution width to platelet count (PDW/PLT); overall survival (OS); nomograph

Submitted Aug 06, 2021. Accepted for publication Oct 19, 2021.

doi: $10.21037 /$ tcr-21-1946

View this article at: https://dx.doi.org/10.21037/tcr-21-1946 


\section{Introduction}

Ovarian cancer ranks fifth in female cancer deaths in the world. Its mortality is higher than that of other reproductive system tumors, ranking first among gynecological malignant tumors. It is reported that there are about 20,000 new cases of ovarian cancer in the world every year (1). The onset of ovarian cancer is hidden, and there is a lack of typical early clinical symptoms and effective early diagnosis methods; as a result, the majority of ovarian cancer patients have reached the advanced stage at their time of diagnosis, and $60-70 \%$ are stage II-IV $(2,3)$. However, the overall prognosis of patients with early diagnosis is good, and their 5-year survival rate is more than $90 \%$ (4). Therefore, early diagnosis of ovarian cancer or timely screening of patients with adverse prognostic characteristics is of great significance.

Prognostic factors of ovarian cancer include age, the International Federation of Gynecology and Obstetrics (FIGO) stage, pathological type, tumor grade, and tumor residue after initial operation (5). Gene detection of ovarian cancer is also a method to predict prognosis, including the detection of BRCA1, BRCA2, CYP1B1, ARID1A, and $p 53$ (6). Although many studies have provided schemes to predict the prognosis of patients with ovarian cancer, the final survival rate has not been further improved (7). Therefore, it is particularly important to find more simple and effective biomarkers to predict the prognosis of patients with ovarian cancer.

The Rho family of GTPase is a small signal G protein family, which mainly plays the role of molecular switch, participates in the regulation of various downstream signal pathways by producing active and inactive GDP binding proteins, and finally participates in various biological events of tumors (8-10). The Rho GTPase activating protein 9 (ARHGAP9) belongs to the Rho GAPase family and is also named RhoGAP9 (11). At present, some studies have reported that the expression of ARHGAP9 is related to the prognosis of various tumors, such as breast cancer (12), gastric cancer (13), bladder cancer (14), hepatocellular carcinoma $(15,16)$, and head and neck squamous cell carcinoma (17). Sun et al. reported for the first time that ginsenoside $\operatorname{Rg} 3$ can effectively inhibit the invasion and migration of hepatocellular carcinoma cells by upregulating the expression of ARHGAP9 protein (16). Based on pathological types, ovarian cancer can be divided into mucinous carcinoma, endometrioid carcinoma, and serous carcinoma, with serous carcinoma accounting for the majority. However, so far, there has been no relevant study reporting the relationship between ARHGAP9 and the prognosis of patients with serous ovarian cancer.

Recent clinical and experimental evidence supports multiple roles of platelets in the progression of malignant tumors. Platelets are a key factor in tumor growth, metastasis, and cancer-related thrombosis (18), including endometrial cancer, gastric cancer, and pancreatic cancer (19). Current studies have found that platelet-related markers, such as platelet to lymphocyte ratio, are more significant prognostic factors in patients with bladder cancer $(20,21)$. Larger platelets store more particles and receptors and stick faster than smaller platelets. Platelet size can more accurately express platelet activity than count (22). The ratio of platelet distribution width to platelet count (PDW/PLT) is a common indicator of platelet size and a surrogate indicator of platelet activation $(23,24)$.

In our preliminary studies, we performed genetic analysis using gene set enrichment analysis (GSEA) and found that the most significant pathway was the ACTIVATION pathway, so this was the key reason for us to study ARHGAP9 in combination with PDW/PLT. This study combined ARHGAP9 and PDW/PLT to evaluate the prognosis of serous ovarian cancer from different perspectives and provided more practical clinical reference for the early diagnosis of serous ovarian cancer or timely screening of patients with adverse prognostic characteristics. We present the following article in accordance with the REMARK reporting checklist (available at https://dx.doi. org/10.21037/tcr-21-1946).

\section{Methods}

\section{Clinical data}

The clinical data of patients with serous ovarian cancer treated in Jiangsu Cancer Hospital from May 2011 to May 2016 were enrolled. The inclusion criteria were as follows: patients (I) diagnosed with ovarian cancer for the first time who underwent surgery; (II) with clinical stage I-III (refer to FIGO stage in 2009); (III) with serous ovarian cancer confirmed by postoperative pathology; (IV) with complete clinical data and follow-up data; (V) who did not receive other antitumor treatment before admission; and (VI) with good compliance. The exclusion criteria were as follows: (I) patients receiving preoperative radiotherapy, chemotherapy, and hormone therapy; (I) patients with other serious diseases, such as severe hypertension; (III) patients with 
some acute diseases, such as infection; and (IV) those who refused follow-up. Finally, 80 patients were included in this study. The age range was 34 to 76 years, and the average age was $50.2 \pm 6.1$ years.

All procedures performed in this study involving human participants were in accordance with the Declaration of Helsinki (as revised in 2013). The study was approved by Jiangsu Cancer Hospital (No. 201048161). Individual consent for this retrospective analysis was waived.

\section{Reverse transcription quantitative polymerase chain reaction (RT-qPCR) metbod for detection of messenger $R N A(m R N A)$ encoding ARHGAP9}

During the operation, the tumor tissues and adjacent tissues were obtained and frozen in a liquid nitrogen tank. The fallopian tube tissues (normal by microscopic examination) were taken as the tumor-adjacent samples. The tumor tissue and adjacent normal tissue samples were taken to a total of $100 \mathrm{mg}$, respectively, ground into powder by liquid nitrogen grinding method, and mixed with $1 \mathrm{~mL}$ Trizol lysate to extract total RNA according to the steps in the manual. The primers were: 5'-CAGAGCAGTGCCTCTCTC-3' (18 bp, $58{ }^{\circ} \mathrm{C}$ ); 5'-CTGCTGGGTCAGATGTCTC-3' (19 bp, $\left.58{ }^{\circ} \mathrm{C}\right)$. Glyceraldehyde 3-phosphate dehydrogenase (GAPDH) were 5'-CATGTTCGTCATGGGTGTGA-3' (20 bp, $\left.60{ }^{\circ} \mathrm{C}\right)$; 5'-ATGGCATGGACTGTGGTCAT-3' $\left(20 \mathrm{bp}, 60^{\circ} \mathrm{C}\right)(14)$. The complementary DNA (cDNA) was obtained by reverse transcription of RNA with reverse transcription kit, and the real-time fluorescence RT-qPCR was performed on the fluorescence qPCR instrument. Finally, the relative expression of the mRNA of the target molecule was calculated by $2^{-\Delta \Delta \mathrm{Ct}}$ method. Based on the median expression, the patients were divided into ARHGAP9 high- and low-expression groups.

\section{Grouping of PDW/PLT high and low groups}

The last preoperative blood routine examination was collected by the medical record management system of our hospital, PDW and PLT in the blood routine were recorded, and then the PDW/PLT ratio was calculated. The receiver operating characteristic (ROC) curve of the relationship between PDW/PLT and postoperative tumor progression was established, and the optimal cut-off value was calculated as 0.0845 . Then, the patients were divided into PDW/PLT high and low groups based on the optimal cut-off value.

\section{Follow-up}

The follow-up methods included outpatient reexamination, readmission medical records, and telephone follow-up. The patients were followed up every 3 months in the first 2 years and every 6 months after the third year. The end point of follow-up was death or follow-up deadline. The deadline for follow-up was May 2021. The survival time was calculated from the start of the follow-up to the end of follow-up or the date of death.

\section{Statistical analysis}

The data were processed using the software SPSS 21.0 (IBM Corp., Armonk, NY, USA). The cut-off value of PDW/ PLT was calculated by ROC curve. The clinicopathological status of the two groups were compared and analyzed using $\chi^{2}$ test. Kaplan-Meier method was used to evaluate the survival of patients, and log-rank statistical method was used to test the significance. Next, the Cox proportional hazards regression model was used to identify the independent prognostic factors that are significant for the prognosis of colorectal cancer patients, and then the nomogram was drawn with $\mathrm{R}$ language (https://www.r-project.org/, Appendix 1) to construct the prediction model. The difference was considered statistically significant when $\mathrm{P}<0.05$. Finally, GSEA was used to explore the possible cellular mechanism of ARHGAP9. Ascites was defined as the volume of abdominal fluid $\geq 200 \mathrm{~mL}$.

\section{Results}

\section{Expression of ARHGAP9 in The Cancer Genome Atlas (TCGA) database}

In TCGA database, 427 serous ovarian cancer tumor tissues and 88 normal ovarian tissues were selected as non-paired comparison samples. It was found that the expression of ARHGAP9 in tumor tissues was higher than that in normal tissues (Figure 1).

\section{Expression of ARHGAP9 in cancer genome atlas}

In TCGA, it was found that among 614 serous ovarian cancer tissues, 260 were low expression and 354 were high 


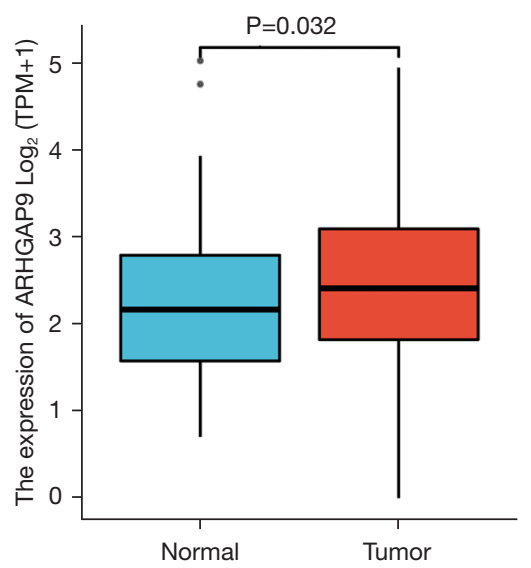

Figure 1 Expression of ARHGAP9 in TCGA database. ARHGAP9, Rho GTPase activating protein 9; TCGA, The Cancer Genome Atlas; TPM, transcripts per million.

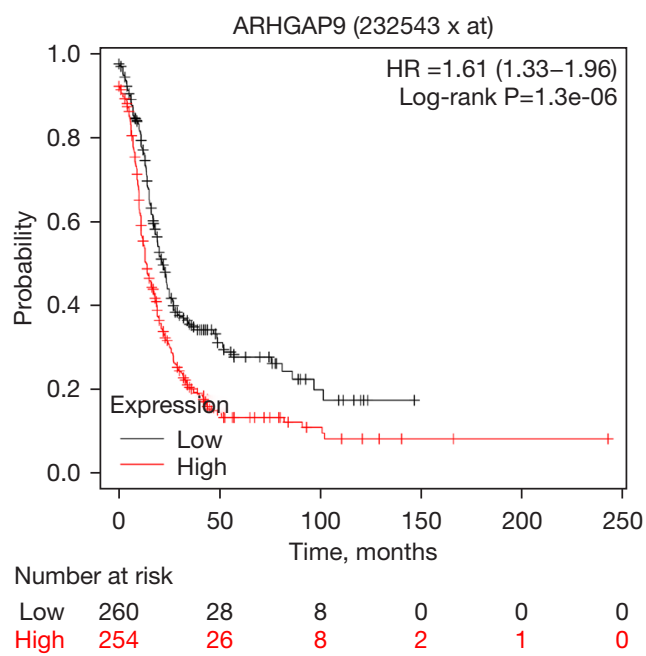

Figure 2 Expression of ARHGAP9 in TCGA. ARHGAP9, Rho GTPase activating protein 9; TCGA, The Cancer Genome Atlas; HR, hazard ratio.

expression, and the prognosis of low expression group was significantly better than that of high expression group $(\mathrm{P}<0.05)$ (Figure 2).

\section{mRNA expression of ARHGAP9 in ovarian cancer tissues}

The tumor tissues of the patient were obtained, and the adjacent fallopian tube (normal by microscopy examination) tissues were taken as the control. The RT-qPCR method was used to detect the mRNA encoding ARHGAP9. It was

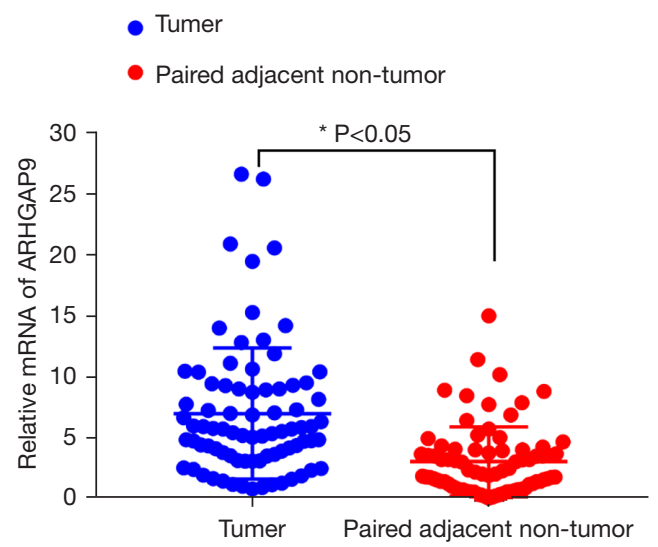

Figure 3 mRNA expression of ARHGAP9 in serous ovarian cancer tissues. mRNA, messenger RNA; ARHGAP9, Rho GTPase activating protein 9 .

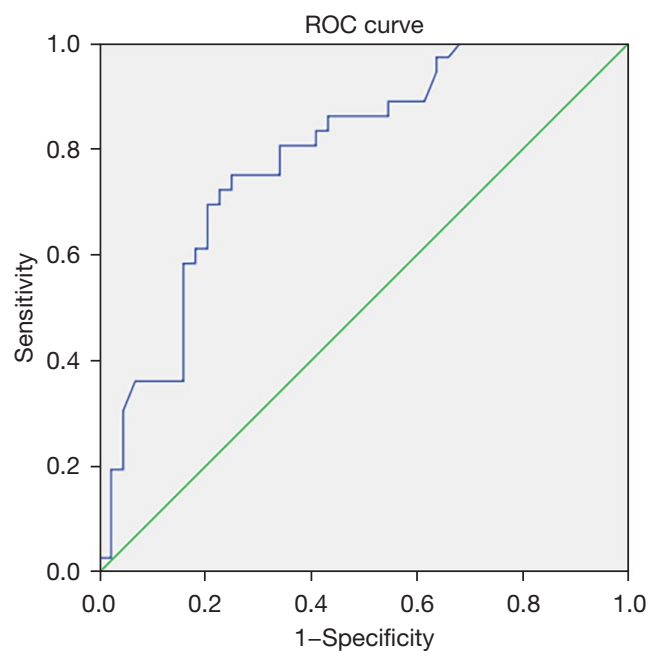

Figure 4 Correlation between PDW/PLT and tumor progression. PDW/PLT, platelet distribution width to platelet count; ROC, receiver operating characteristic.

found that ARHGAP9 was highly expressed in the cancer tissues (Figure 3).

\section{Correlation between PDW/PLT and tumor progression}

The ROC curve of the correlation between preoperative $\mathrm{PDW} / \mathrm{PLT}$ and tumor progression in 80 patients with serous ovarian cancer is shown in Figure 4. The optimal cutoff value of PDW/PLT was 0.0845 [area under the curve $(\mathrm{AUC})=0.789, \mathrm{P}=0.000]$. 

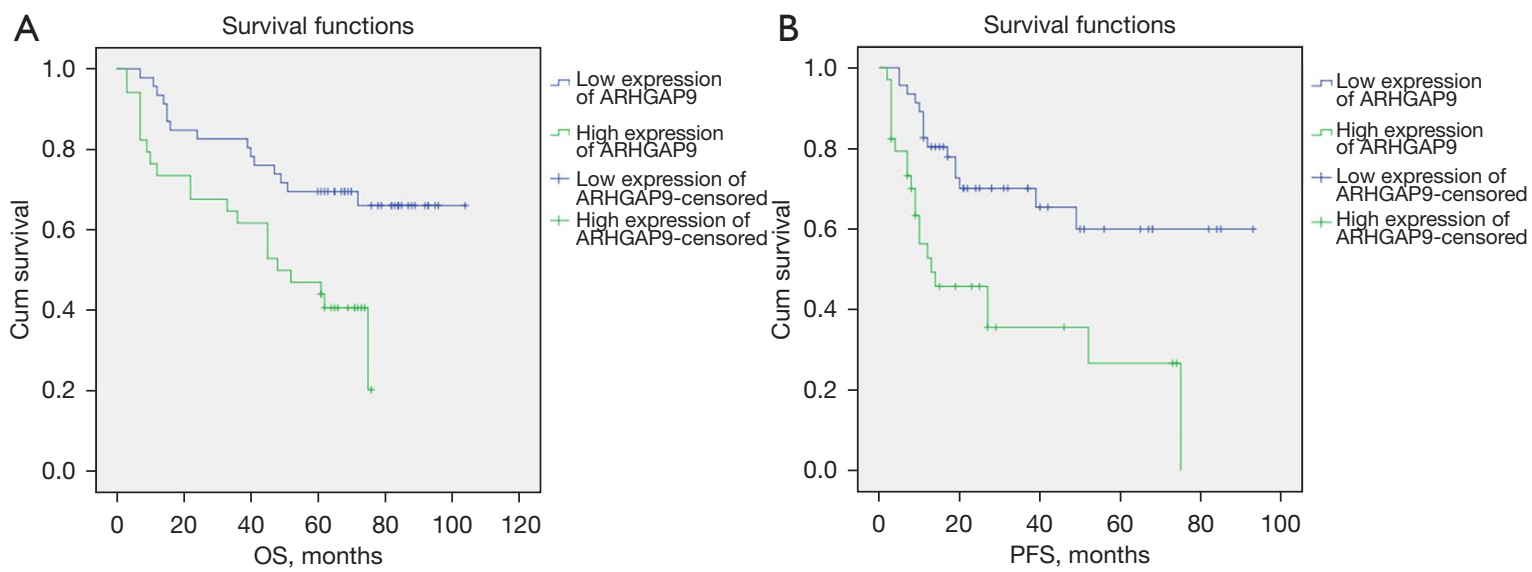

Figure 5 OS and PFS of ARHGAP9 high and low expression groups. OS, overall survival; PFS, progression-free survival; ARHGAP9, Rho GTPase activating protein 9.
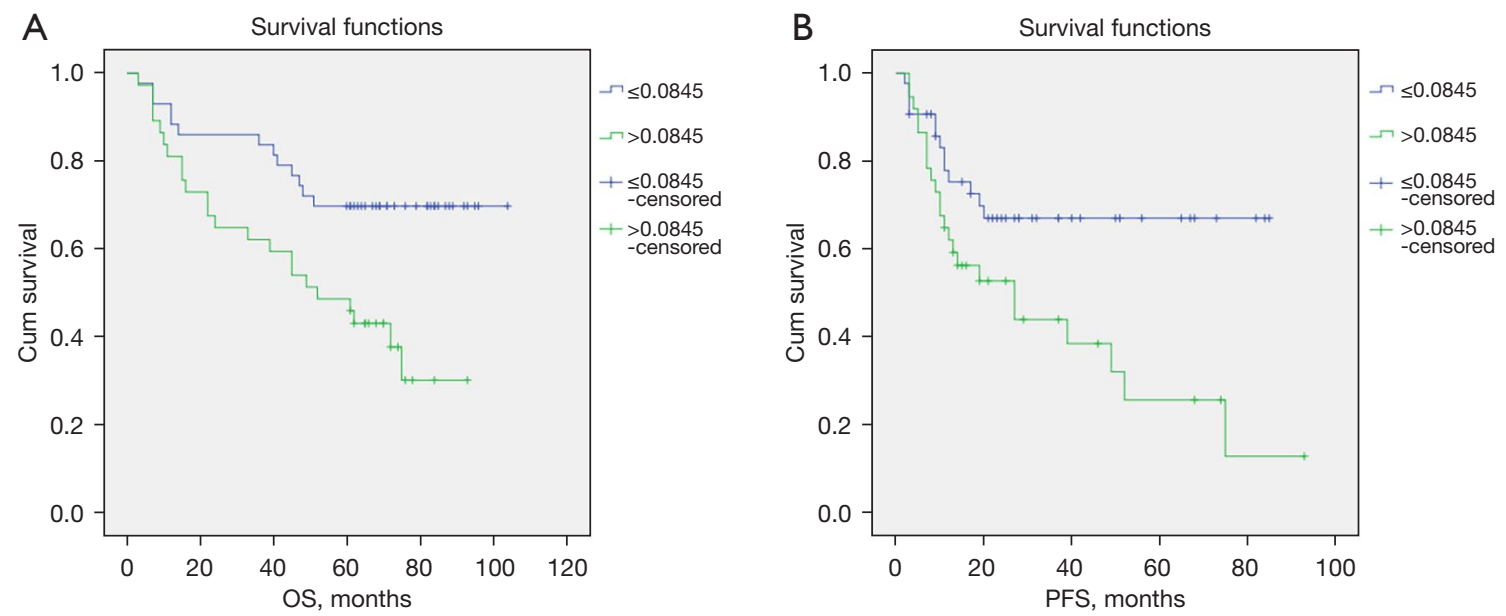

Figure 6 OS and PFS of high and low PDW/PLT groups. OS, overall survival; PFS, progression-free survival; PDW/PLT, platelet distribution width to platelet count.

\section{Comparison of overall survival (OS) and progression-free survival (PFS) between the two groups}

Finally, 80 patients were included in the study, among whom 36 participants died, the mortality rate was $45.0 \%$; a total of 44 participants survived, the survival rate was $55.0 \%$; the median PFS was 19 months and the median OS was 62.5 months. The OS and PFS of the ARHGAP9 low expression group and low PDW/PLT group were significantly better than those of the ARHGAP9 high expression group and high PDW/PLT group (all $\mathrm{P}<0.05)$ (Figures 5,6).

\section{Relationship between ARHGAP9, PDW/PLT, and clinicopatbological data}

It was found that ARHGAP9 is highly expressed in TCGA serous ovarian cancer database and the serous ovarian cancer tumor tissues. There were significant differences in menstrual status, FIGO stage, and grade between the ARHGAP9 low- and high-expression groups (all $\mathrm{P}<0.05)$. There were significant differences in FIGO stage, lymph node metastasis, and ascites between low PDW/PLT and high PDW/PLT groups (all $\mathrm{P}<0.05)$ (Table 1). 
Table 1 Relationship between ARHGAP9, PDW/PLT and clinical data

\begin{tabular}{|c|c|c|c|c|c|c|c|}
\hline Variables & $\begin{array}{c}\text { Total } \\
(\mathrm{n}=80)\end{array}$ & \multicolumn{3}{|c|}{ ARHGAP9 } & \multicolumn{3}{|c|}{ PDW/PLT } \\
\hline$\leq 50$ & 70 & 37 & 33 & & 37 & 33 & \\
\hline Menopausal & 34 & 10 & 24 & & 14 & 20 & \\
\hline Premenopausal & 46 & 36 & 10 & & 29 & 17 & \\
\hline FIGO stage & & & & $0.009^{*}$ & & & $0.006^{*}$ \\
\hline Yes & 11 & 5 & 6 & & 10 & 1 & \\
\hline No & 69 & 41 & 28 & & 33 & 36 & \\
\hline Grade & & & & $0.001^{*}$ & & & 0.271 \\
\hline Low level & 10 & 1 & 9 & & 7 & 3 & \\
\hline High level & 70 & 45 & 25 & & 36 & 34 & \\
\hline Ascites or not & & & & 0.384 & & & $0.008^{*}$ \\
\hline Yes & 11 & 5 & 6 & & 10 & 1 & \\
\hline
\end{tabular}

*, $\mathrm{P}<0.05$, the difference is statistically significant. ARHGAP9, Rho GTPase activating protein 9; PDW/PLT, platelet distribution width to platelet count; FIGO, International Federation of Gynecology and Obstetrics.

\section{Results of univariate analysis}

Univariate analysis showed that PDW/PLT and ARHGAP9 were the factors affecting OS (both $\mathrm{P}<0.05$ ) (Table 2).

\section{Results of multivariate analysis}

Cox multivariate analysis showed that PDW/PLT and ARHGAP9 were independent risk factors affecting tumor progression $(\mathrm{P}=0.026, \mathrm{P}=0.028)$ (Table 3).

\section{Nomogram drawing by $R$ language and prediction model construction}

In internal validation, the $\mathrm{C}$-index of nomogram was 0.6518 [95\% confidence interval (CI): 0.5685 to 0.7352 ], and the prediction model had certain accuracy (Figure 7).

\section{Correlation between ARHGAP9 and bomologous recombination repair deficiency-related genes}

We also found that ARHGAP9 had a good correlation with homologous recombination repair deficiency related genes (ATM, BRCA2, MRE11A, RAD51D) $(\mathrm{P}<0.05)$ (Figure 8).

\section{Correlation between ARHGAP9 and tumor immune infiltration}

The correlation between ARHGAP9 and tumor immune infiltration was discussed by using the relevant modules of Cell-type Identification by Estimating Relative Subsets of RNA Transcripts (CIBERSORT) and Gene Expression Profiling Interactive Analysis (GEPIA). The data analysis of CIBERPORT and GEPIA showed that ARHGAP9 was correlated with 24 immune cells such as T cells, interstitial 
Table 2 Univariate analysis of clinical factors on OS

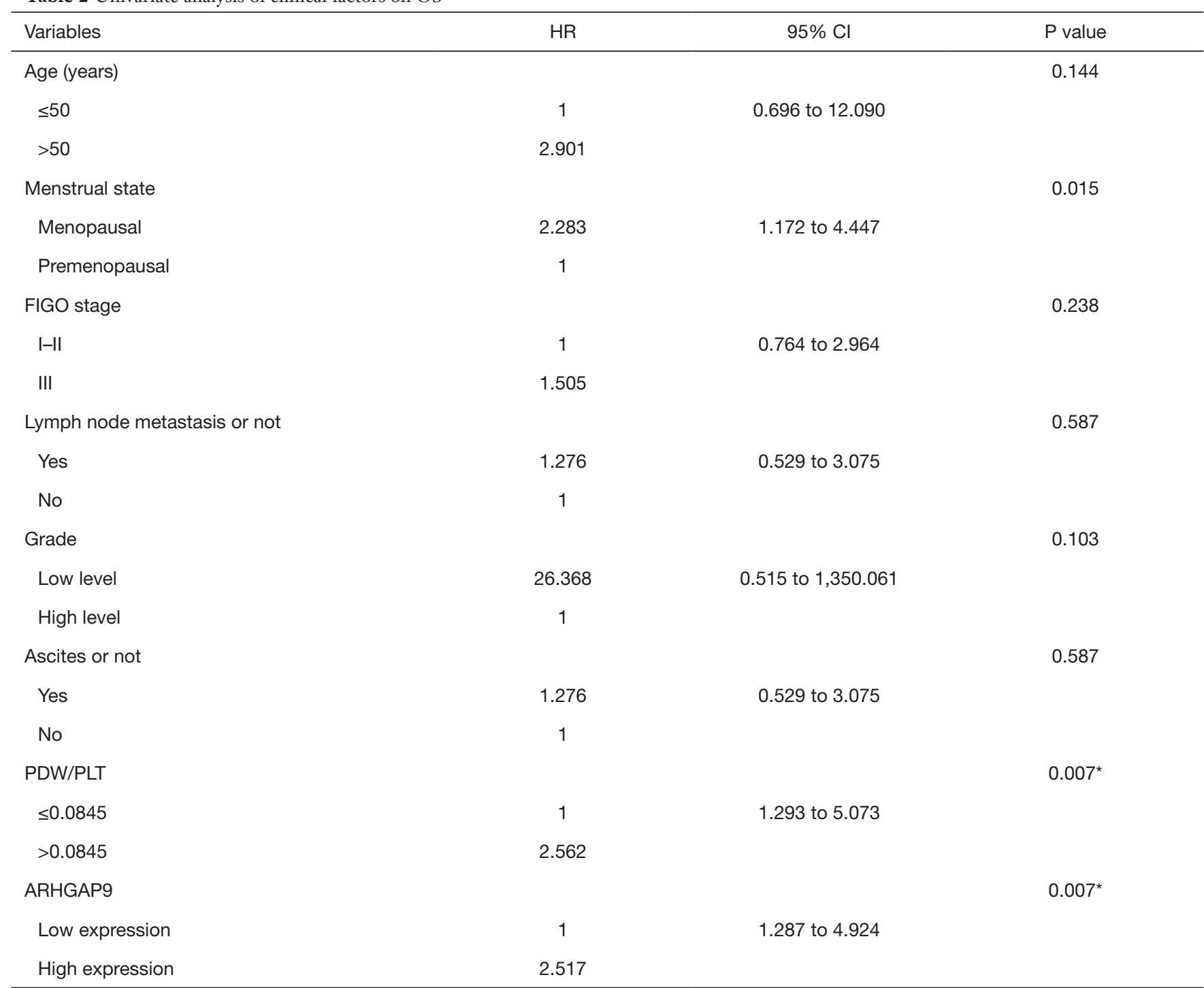

* $\mathrm{P}<0.05$, the difference is statistically significant. OS, overall survival; HR, hazard ratio; $\mathrm{Cl}$, confidence interval; FIGO, International Federation of Gynecology and Obstetrics; PDW/PLT, platelet distribution width to platelet count; ARHGAP9, Rho GTPase activating protein 9.

dendritic cells (iDC), T helper (Th)1 cells, and regulatory T cells (Treg) (Figure 9), in which Treg was one of the main immune cells affected by ARHGAP9 expression (Figure 10).

\section{GSEA results}

Based on the GSEA analysis results, eight statistically significant possible related pathways were obtained, including: (I) platelet activation; (II) immune cells and microRNAs in tumor microenvironment; (III) DNA repair;
(IV) involved in megakaryocyte differentiation and platelet function; (V) cancer immunotherapy by PD-1 blockade; (VI) DNA double strand break repair; (VII) Wnt; (VIII) T cell signal transduction (Figure 11).

\section{Discussion}

Ovarian cancer is a malignant tumor of the female reproductive system, and its incidence is only secondary to that of cervical cancer and endometrial cancer (25). Its 
Table 3 Multivariate analysis of clinical factors on OS

\begin{tabular}{lccc}
\hline Variables & HR & $95 \% \mathrm{Cl}$ & P value \\
\hline PDW/PLT & 1 & 1.098 to 4.420 & \\
$\leq 0.0845$ & 2.203 & & $0.028^{\star}$ \\
$>0.0845$ & & & 1.086 to 4.255 \\
ARHGAP9 & 1 & & \\
Low expression & 2.150 & & \\
High expression & & \\
\hline
\end{tabular}

*, $\mathrm{P}<0.05$, the difference is statistically significant. OS, overall survival; HR, hazard ratio; Cl, confidence interval; PDW/PLT, platelet distribution width to platelet count; ARHGAP9, Rho GTPase activating protein 9.

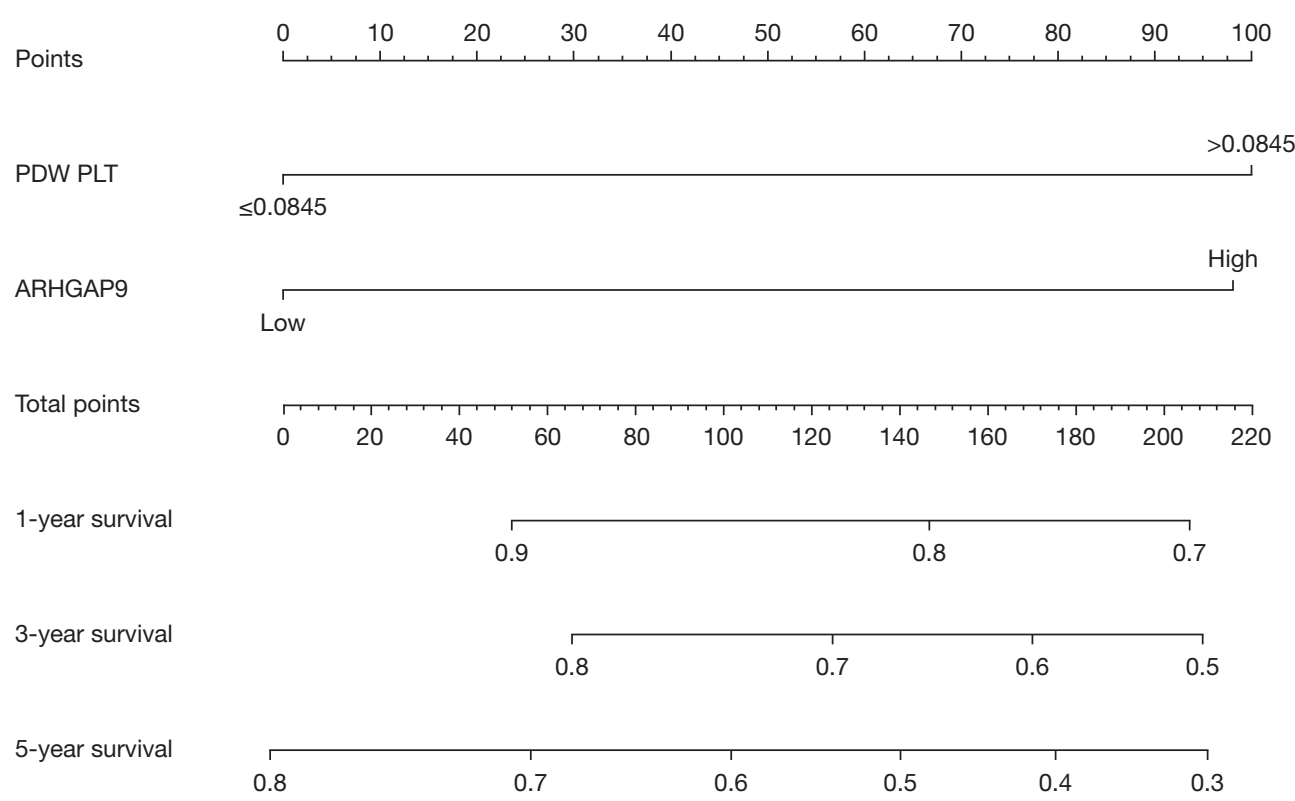

Figure 7 Nomogram prediction model. PDW/PLT, platelet distribution width to platelet count; ARHGAP9, Rho GTPase activating protein 9 .

recurrence rate is very high. Serous ovarian cancer is one of the most common pathological types. Therefore, for the early diagnosis of serous ovarian cancer, it is of great positive significance to promptly screen patients with adverse prognostic characteristics or develop targeted drugs with therapeutic significance. In our study, we showed that ARHGAP9 and preoperative PDW/PLT are related to the prognosis of patients with ovarian cancer, and the reduction of ARHGAP9 and PDW/PLT can significantly prolong the OS and PFS of such patients. At the same time, ARHGAP9 may also be related to the immune infiltration of ovarian cancer.
The tumor microenvironment is a pathological environment composed of tumor cells, stromal cells, cytokines, and immune cells, playing an important role in the occurrence and progression of ovarian cancer; many studies have confirmed that immune and inflammatory responses may be the key factors in the progression of ovarian cancer (26). A member of RhoGAP family, ARHGAP9, is composed of amino terminal SH3 domain, WW domain, pleckstrin homology domain, and carboxyl terminal RhoGAP domain (11). It can inactivate Rho GTPases and inhibit a variety of cellular processes, such as gene transcription, cell proliferation, cell migration, 

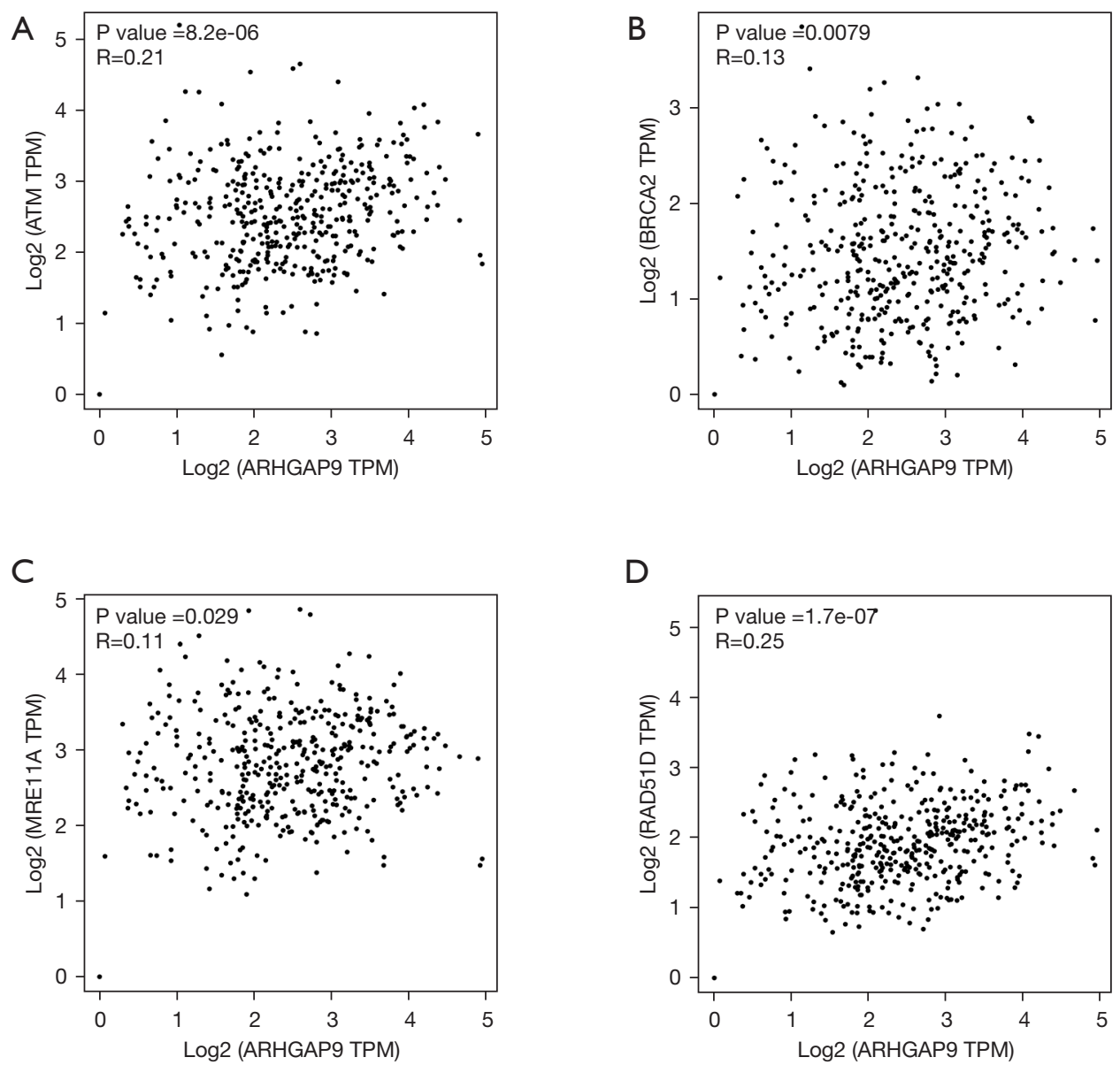

Figure 8 Correlation between ARHGAP9 and homologous recombination repair deficiency related genes (ATM, BRCA2, MRE11A, RAD51D). ARHGAP9, Rho GTPase activating protein 9; TPM, transcripts per million.

and cell invasion, by promoting the hydrolysis of GTPbound Rho GTPases (27). At present, many studies have reported that ARHGAP9 is involved in various biological events of various tumors. Wang et al. (12) found that the decreased expression of ARHGAP9 could inhibit the proliferation, migration, and invasion of breast cancer. Sun et al. (13) found that ARHGAP9 small interfering RNA (siRNA) inhibits the proliferation of gastric cancer cells and epithelial-mesenchymal transition (EMT) by inactivating Akt and p38 signal transduction and inhibiting MMP2 and MMP9. However, so far, there has been no relevant study on the relationship between ARHGAP9 and the prognosis of patients with serous ovarian cancer.

Accumulating evidence shows that inflammation can play an important role in the occurrence and development of tumors by promoting tumor angiogenesis and tumor cell proliferation and inhibiting tumor cell apoptosis (28).
Although there have been many recent studies on the clinical significance of activated platelets in cancer, the scope of available data is still limited by the type of malignant tumor and the clinical results studied. Platelets are rich in growth factors, such as transforming growth factor- $\beta$; these platelet-derived growth factors are usually produced in large quantities by cancer cells and contribute to their development (29). The PDW is a more specific marker of platelet activation because it does not increase due to platelet swelling (30), and it is also a method to measure platelet heterogeneity caused by megakaryocyte heterogeneity (31). It has been reported that higher PDW is associated with poor prognosis of ovarian cancer (32). In the tumor microenvironment, the tumor tissue produces chemokines, and the platelets in the blood enter the tumor tissue through the blood vessel wall under the action of chemokines. These infiltrated platelets are called tumor-associated platelets 


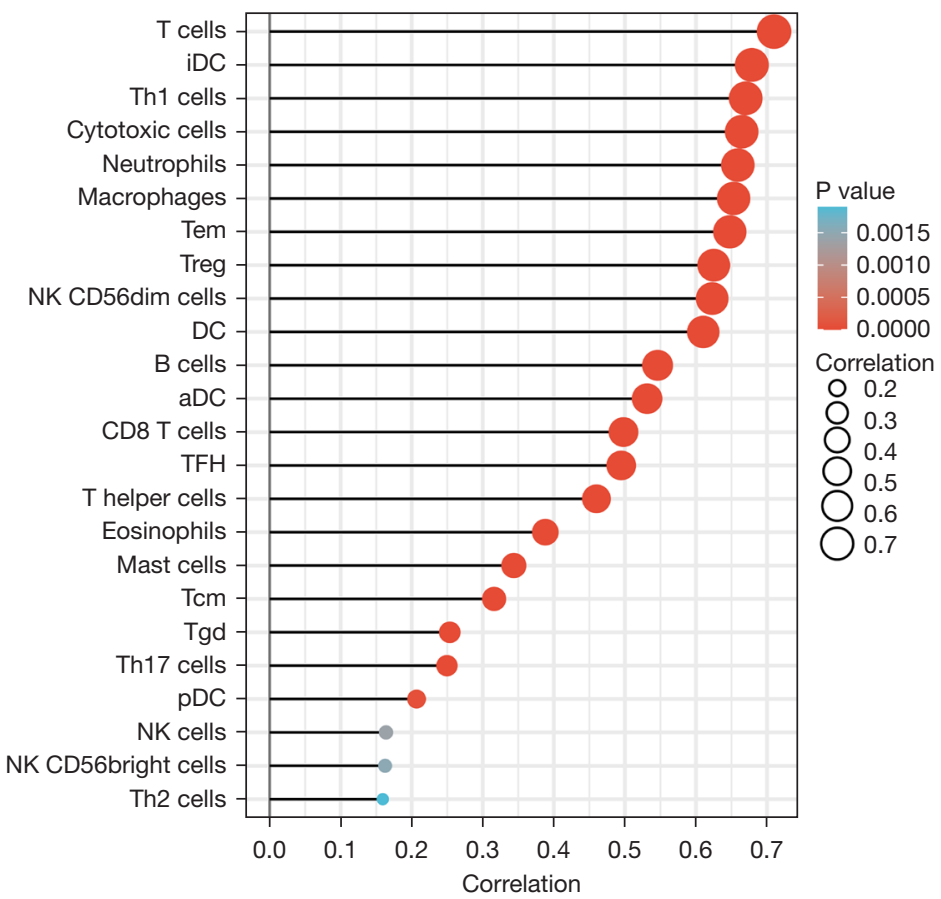

Figure 9 Correlation between ARHGAP9 and 24 immune cells. ARHGAP9, Rho GTPase activating protein 9.

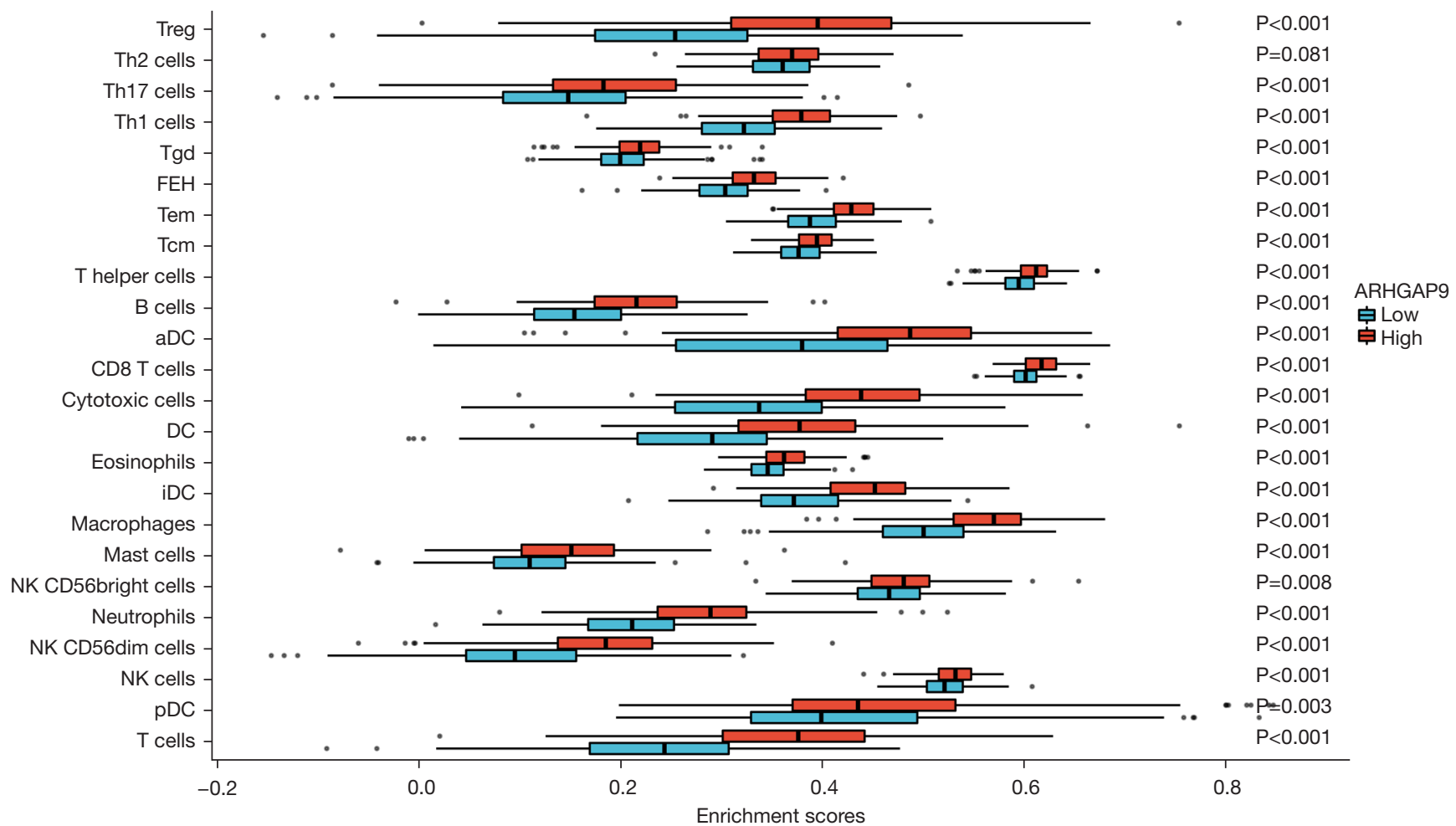

Figure 10 Correlation between ARHGAP9 high or low expression groups and 24 immune cells. ARHGAP9, Rho GTPase activating protein 9 . 

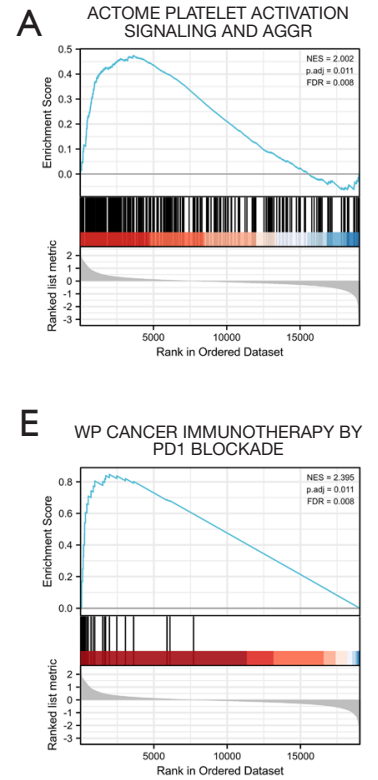
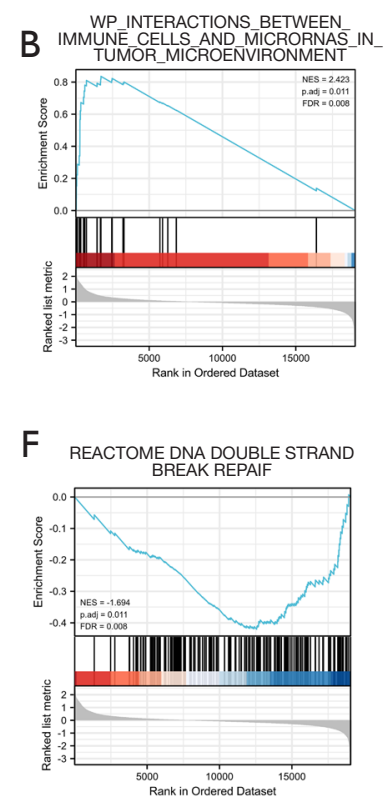
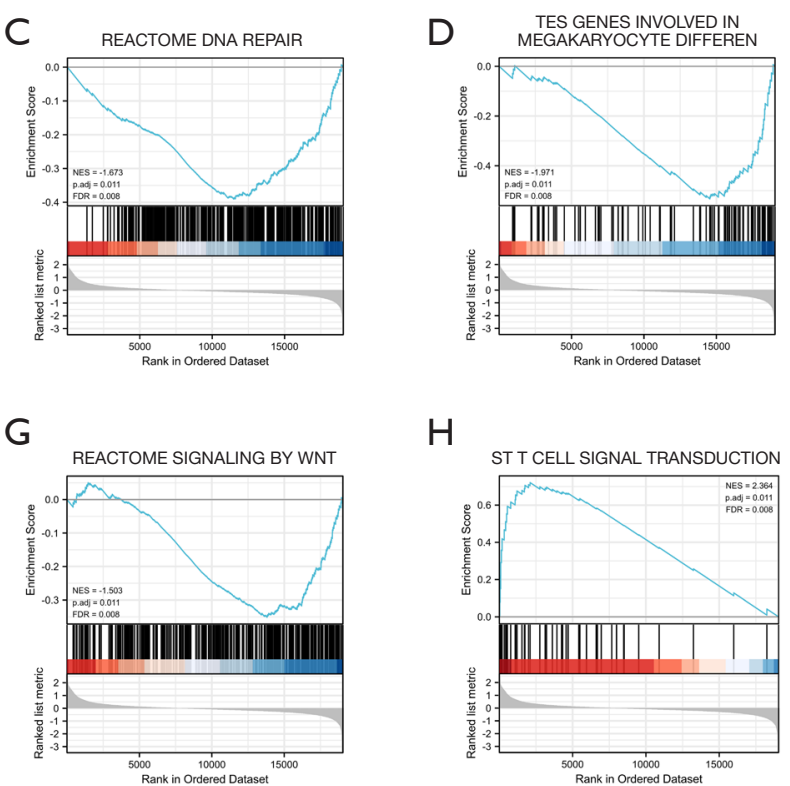

$\mathrm{H}$

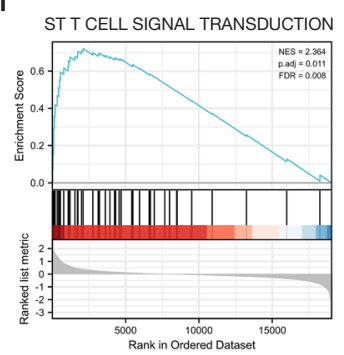

Figure 11 The eight significant possible related pathways. A gene set from MSigDB was used for the biological process of otobiology. One thousand random samples were arranged. NES, normalized enrichment score; FDR, false discovery rate.

(TAP) (33). In recent years, studies have increasingly shown that TAP induced under tumor conditions plays a very important role in the occurrence and development of tumors $(34,35)$. Therefore, taking the ratio of PDW to PLT as an indicator to evaluate the inflammatory state of patients has more relevant clinical significance.

This study combined ARHGAP9 and PDW/PLT to evaluate the prognosis of serous ovarian cancer from different angles. This topic is significant from the perspective of tumor microenvironment balance. In our study, we first described the expression of ARHGAP9 in serous ovarian cancer and TCGA and showed that it has the characteristics of cancer promoting genes. Moreover, we used RT-qPCR to detect the expression of the mRNA encoding ARHGAP9 in serous ovarian cancer tissues. It was found that ARHGAP9 was also highly expressed in our serous ovarian cancer tissues, which was consistent with the expression in the database. There were significant differences in menstrual status, FIGO stage and ascites between ARHGAP9 low expression group and ARHGAP9 high expression group (all $\mathrm{P}<0.05)$. There were significant differences in FIGO stage, lymph node metastasis, and ascites between the low PDW/PLT group and high PDW/PLT group (all $\mathrm{P}<0.05$ ). Finally, 80 patients were included in the study, among whom 36 participants died, the mortality rate was $45.0 \%$; a total of 44 participants survived, the survival rate was $55.0 \%$; the median PFS was 19 months, and the median OS was 62.5 months. At the end of follow-up, it was found that OS and PFS in the ARHGAP9 low expression group and low PDW/PLT group were significantly better than those in ARHGAP9 high expression group and high PDW/PLT group (all $\mathrm{P}<0.05)$. Cox multivariate analysis also showed that PDW/ PLT and ARHGAP9 were independent risk factors for tumor progression $(\mathrm{P}=0.026$ and $\mathrm{P}=0.028$, respectively). This result also reflects the positive significance of our research on selecting such a joint indicator. To provide a better reference for the clinical management of patients with serous ovarian cancer, we constructed a prediction model based on multivariate analysis. The C-index of the nomogram was 0.6518 (95\% CI: 0.5685 to 0.7352 ), which also showed that the prediction model has certain accuracy. The recurrence rate of serous ovarian cancer is high, and platinum-containing chemotherapy is often the first choice after recurrence. However, not all patients can be assigned to the benefited group; therefore, the recommended targeted therapy is also in the optional range. The guidelines on targeted treatment of ovarian cancer recommend that patients with stage II-IV do not use bevacizumab for first-line treatment, and olaparib is recommended for maintenance treatment as long as patients with $B R C A$ mutation exist, no matter complete remission 
or partial remission (36,37); bevacizumab is used for firstline treatment in patients with stage II-IV, and bevacizumab can be selected for maintenance treatment, regardless of complete remission or partial remission, and olaparib can be selected for patients with BRCA mutation $(38,39)$. Since ovarian cancer patients are prone to homologous recombination repair deficiency, we further conducted relevant analysis and detection. We found that ARHGAP9 has a good correlation with homologous recombination repair deficiency related genes (ATM, BRCA2, MRE11A, $R A D 51 D)$. Therefore, from a certain point of view, we can speculate that patients with high expression of ARHGAP9 and poor postoperative adjuvant chemotherapy effect may have deficiency in homologous recombination repair, such as BRCA2 mutation. Tumor-infiltrating lymphocytes are independent predictors of sentinel lymph node status and survival in cancer patients (40). Therefore, we tried to determine whether the expression of ARHGAP9 is related to the immune infiltration of ovarian cancer. Analysis of CIBERSORT and GEPIA data showed that ARHGAP9 was correlated with 24 immune cells such as T cells, iDC, Th1 cells, and Treg, among which Treg was one of the main immune cells affected by ARHGAP9 expression. Treg is a kind of $T$ cell subset that controls autoimmunity in vivo and is one of the important factors to maintain immune tolerance. At present, it has been reported that Treg cell population in peripheral blood of patients with ovarian cancer is related to long-term prognosis (41). Therefore, we can boldly speculate that the expression of ARHGAP9 is related to the immune infiltration of ovarian cancer. Chen et al. (42) found that PD-1/PD-L1 signaling pathway has a certain regulatory effect on Treg in ovarian cancer. Therefore, from a certain point of view, it can be considered that for ovarian cancer patients with high expression of ARHGAP9, the use of PD-1/PD-L1 inhibitors such as pabolizumab and sintilimab may have certain therapeutic significance. Of course, these findings need to be verified by further clinical trials. Finally, we used GSEA to explore the possible cellular mechanism and obtained eight statistically significant possible related pathways, which were: (I) platelet activation; (II) immune cells and microRNAS in tumor microenvironment; (III) DNA repair; (IV) involved in megakaryocyte differentiation and platelet function; (V) cancer immunotherapy by PD-1 blockade; (VI) DNA double strand break repair; (VII) Wnt; (VIII) T cell signal transduction. It can be found that these possible pathways include platelet activation, DNA repair and damage, and are related to $\mathrm{PD}-1$, which again demonstrates the clinical and scientific significance of ARHGAP9 molecule in serous ovarian cancer, which lays a certain foundation for later basic experiments.

\section{Conclusions}

Despite some limitations of this study, the data clearly show that the reduction of ARHGAP9 and PDW/PLT can significantly prolong OS and PFS in patients with serous ovarian cancer. Further multicenter, large sample, prospective studies are necessary to clarify the exact governing mechanism of ARHGAP9 and PDW/PLT in serous ovarian cancer.

\section{Acknowledgments}

Funding: None.

\section{Footnote}

Reporting Checklist: The authors have completed the REMARK reporting checklist. Available at https://dx.doi. org/10.21037/tcr-21-1946

Data Sharing Statement: Available at https://dx.doi. org/10.21037/tcr-21-1946

Conflicts of Interest: All authors have completed the ICMJE uniform disclosure form (available at https://dx.doi. org/10.21037/tcr-21-1946). The authors have no conflicts of interest to declare.

Ethical Statement: The authors are accountable for all aspects of the work in ensuring that questions related to the accuracy or integrity of any part of the work are appropriately investigated and resolved. All procedures performed in this study involving human participants were in accordance with the Declaration of Helsinki (as revised in 2013). The study was approved by Jiangsu Cancer Hospital (No. 201048161). Individual consent for this retrospective analysis was waived.

Open Access Statement: This is an Open Access article distributed in accordance with the Creative Commons Attribution-NonCommercial-NoDerivs 4.0 International License (CC BY-NC-ND 4.0), which permits the noncommercial replication and distribution of the article with the strict proviso that no changes or edits are made and the 
original work is properly cited (including links to both the formal publication through the relevant DOI and the license). See: https://creativecommons.org/licenses/by-nc-nd/4.0/.

\section{References}

1. Siegel RL, Miller KD, Fuchs HE, et al. Cancer statistics, 2021. CA Cancer J Clin 2021;71:7-33.

2. Hulstaert E, Morlion A, Levanon K, et al. Candidate RNA biomarkers in biofluids for early diagnosis of ovarian cancer: a systematic review. Gynecol Oncol 2021;160:633-42.

3. Zhang $\mathrm{Y}$, Wang $\mathrm{X}$, Chen $\mathrm{X}$. Identification of core genes for early diagnosis and the EMT modulation of ovarian serous cancer by bioinformatics perspective. Aging (Albany NY) 2021;13:3112-45.

4. Pauler DK, Menon U, McIntosh M, et al. Factors influencing serum CA125II levels in healthy postmenopausal women. Cancer Epidemiol Biomarkers Prev 2001;10:489-93.

5. Gadducci A, Cosio S, Tana R, et al. Serum and tissue biomarkers as predictive and prognostic variables in epithelial ovarian cancer. Crit Rev Oncol Hematol 2009;69:12-27.

6. Miki Y, Swensen J, Shattuck-Eidens D, et al. A strong candidate for the breast and ovarian cancer susceptibility gene BRCA1. Science 1994;266:66-71.

7. Aboutalebi H, Bahrami A, Soleimani A, et al. The diagnostic, prognostic and therapeutic potential of circulating microRNAs in ovarian cancer. Int $\mathrm{J}$ Biochem Cell Biol 2020;124:105765.

8. Heasman SJ, Ridley AJ. Mammalian Rho GTPases: new insights into their functions from in vivo studies. Nat Rev Mol Cell Biol 2008;9:690-701.

9. Amin E, Jaiswal M, Derewenda U, et al. Deciphering the molecular and functional basis of RHOGAP family proteins: a systematic approach toward selective inactivation of Rho family proteins. J Biol Chem 2016;291:20353-71.

10. Humphries BA, Wang $Z$, Yang C. MicroRNA regulation of the small Rho GTPase regulators-complexities and opportunities in targeting cancer metastasis. Cancers (Basel) 2020;12:1092.

11. Furukawa Y, Kawasoe T, Daigo Y, et al. Isolation of a novel human gene, ARHGAP9, encoding a rho-GTPase activating protein. Biochem Biophys Res Commun 2001;284:643-9.

12. Wang T, Ha M. Silencing ARHGAP9 correlates with the risk of breast cancer and inhibits the proliferation, migration, and invasion of breast cancer. J Cell Biochem 2018;119:7747-56.

13. Sun L, Zhang Y, Lou J. ARHGAP9 siRNA inhibits gastric cancer cell proliferation and EMT via inactivating Akt, p38 signaling and inhibiting MMP2 and MMP9. Int J Clin Exp Pathol 2017;10:11979-85.

14. Piao XM, Jeong P, Yan C, et al. A novel tumor suppressing gene, ARHGAP9, is an independent prognostic biomarker for bladder cancer. Oncol Lett 2020;19:476-86.

15. Zhang H, Tang QF, Sun MY, et al. ARHGAP9 suppresses the migration and invasion of hepatocellular carcinoma cells through up-regulating FOXJ2/E-cadherin. Cell Death Dis 2018;9:916.

16. Sun MY, Song YN, Zhang M, et al. Ginsenoside Rg3 inhibits the migration and invasion of liver cancer cells by increasing the protein expression of ARHGAP9. Oncol Lett 2019;17:965-73.

17. Song Y, Pan Y, Liu J. The relevance between the immune response-related gene module and clinical traits in head and neck squamous cell carcinoma. Cancer Manag Res 2019;11:7455-72.

18. Mege D, Aubert M, Lacroix R, et al. Involvement of platelets in cancers. Semin Thromb Hemost 2019;45:569-75.

19. Suzuki K, Aiura K, Kitagou M, et al. Platelets counts closely correlate with the disease-free survival interval of pancreatic cancer patients. Hepatogastroenterology 2004;51:847-53.

20. Gasparyan AY, Ayvazyan L, Mikhailidis DP, et al. Mean platelet volume: a link between thrombosis and inflammation? Curr Pharm Des 2011;17:47-58.

21. Ekici H, Malatyalioglu E, Kokcu A, et al. Do leukocyte and platelet counts have benefit for \preoperative evaluation of endometrial cancer? Asian Pac J Cancer Prev 2015;16:5305-10.

22. Bardyn M, Rappaz B, Jaferzadeh K, et al. Red blood cells ageing markers: a multi-parametric analysis. Blood Transfus 2017;15:239-48.

23. Martin JF, Bath PM, Burr ML. Influence of platelet size on outcome after myocardial infarction. Lancet 1991;338:1409-11.

24. Pozza A, Pauletti B, Scarpa M, et al. Prognostic role of neutrophil-to-lymphocyte ratio and platelet-tolymphocyte ratio in patients with midgut neuroendocrine tumors undergoing resective surgery. Int J Colorectal Dis 2019;34:1849-56.

25. DeVorkin L, Hattersley M, Kim P, et al. Autophagy inhibition enhances sunitinib efficacy in clear cell ovarian 
carcinoma. Mol Cancer Res 2017;15:250-8.

26. Browning L, Patel MR, Horvath EB, et al. IL-6 and ovarian cancer: inflammatory cytokines in promotion of metastasis. Cancer Manag Res 2018;10:6685-93.

27. Tcherkezian J, Lamarche-Vane N. Current knowledge of the large RhoGAP family of proteins. Biol Cell 2007;99:67-86.

28. Moses K, Brandau S. Human neutrophils: Their role in cancer and relation to myeloid-derived suppressor cells. Semin Immunol 2016;28:187-96.

29. Miyazono K, Yuki K, Takaku F, et al. Latent forms of TGF-beta: structure and biology. Ann N Y Acad Sci 1990;593:51-8.

30. Vagdatli E, Gounari E, Lazaridou E, et al. Platelet distribution width: a simple, practical and specific marker of activation of coagulation. Hippokratia 2010;14:28-32.

31. Paulus JM. Recent advances in the story of megakaryocyte physiology. Pathol Biol (Paris) 1981;29:133-5.

32. Qin L, Li JY, Huang WJ, et al. Higher platelet distribution width is associated with unfavorable prognosis in ovarian cancer. Cancer Biomark 2020;28:365-70.

33. Dymicka-Piekarska V, Koper-Lenkiewicz OM, Zińczuk $\mathrm{J}$, et al. Inflammatory cell-associated tumors. Not only macrophages (TAMs), fibroblasts (TAFs) and neutrophils (TANs) can infiltrate the tumor microenvironment. The unique role of tumor associated platelets (TAPs). Cancer Immunol Immunother 2021;70:1497-510.

34. Amirkhosravi A, Amaya M, Siddiqui F, et al. Blockade of GpIIb/IIIa inhibits the release of vascular endothelial growth factor (VEGF) from tumor cell-activated platelets and experimental metastasis. Platelets 1999;10:285-92.

35. Xu Y, Liu J, Liu Z, et al. Blockade of platelets using tumor-specific NO-releasing nanoparticles prevents tumor metastasis and reverses tumor immunosuppression. ACS Nano 2020;14:9780-95.

36. Lv X, Cui S, Zhang X, et al. Efficacy and safety of neoadjuvant chemotherapy versus primary debulking surgery in patients with ovarian cancer: a meta-analysis. J Gynecol Oncol 2020;31:e12.

37. Vincent L, Jankowski C, Ouldamer L, et al. Prognostic factors of overall survival for patients with FIGO stage IIIc or IVa ovarian cancer treated with neo-adjuvant chemotherapy followed by interval debulking surgery: a multicenter cohort analysis from the FRANCOGYN study group. Eur J Surg Oncol 2020;46:1689-96.

38. Armstrong DK, Alvarez RD, Bakkum-Gamez JN, et al. Ovarian cancer, version 2.2020, NCCN clinical practice guidelines in oncology. J Natl Compr Canc Netw 2021;19:191-226.

39. Doddato G, Valentino F, Giliberti A, et al. Whole exome sequencing in BRCA1-2 candidate families: the contribution of other cancer susceptibility genes. Front Oncol 2021;11:649435.

40. Azimi F, Scolyer RA, Rumcheva P, et al. Tumor-infiltrating lymphocyte grade is an independent predictor of sentinel lymph node status and survival in patients with cutaneous melanoma. J Clin Oncol 2012;30:2678-83.

41. Dutsch-Wicherek MM, Szubert S, Dziobek K, et al. Analysis of the treg cell population in the peripheral blood of ovarian cancer patients in relation to the long-term outcomes. Ginekol Pol 2019;90:179-84.

42. Chen JX, Yi XJ, Gao SX, et al. The possible regulatory effect of the PD-1/PD-L1 signaling pathway on Tregs in ovarian cancer. Gen Physiol Biophys 2020;39:319-30.

(English Language Editor: J. Jones)
Cite this article as: Shen $\mathrm{Y}, \mathrm{Xu} \mathrm{H}$, Guan Z, Lv M, Qian T, Wu Y. Effect of Rho GTPase activating protein 9 combined with preoperative ratio of platelet distribution width to platelet count on prognosis of patients with serous ovarian cancer. Transl Cancer Res 2021;10(10):4440-4453. doi: 10.21037/tcr21-1946 


\section{Supplementary}

setwd("C:/Users/wjc24/Desktop/aa ")

\# Loading the Installation Package

library(rms)

library(foreign)

library(survival)

\# reading data

tcga<-read.table("clinical.txt",header=T,sep="\t")

\# Convert data to factor format

tcga\$ Grade<-factor(tcga\$ Grade,labels=c("G3"," G1+G2"))

tcga\$ PDW_PLT <-factor(tcga\$ PDW_PLT,labels=c(">0.0845"," $\leq 0.0845 "))$

tcga $\$$ ARHGAP9<-factor(tcga $\$$ ARHGAP9, labels=c("low "," high "))

\# Pack your data

ddist $<$ - datadist(tcga)

options(datadist='ddist')

\# Construct the multivariate Cox regression model

cox $<-\operatorname{cph}($ Surv(survival_time,status) Grade + PDW_PLT + ARHGAP9,surv=T,x=T, y=T,data=tcga)

surv $<-$ Survival(cox)

surv <- Survival(cox)

sur_3_year<-function(x)surv $(1 * 12 * 3, \mathrm{lp}=\mathrm{x}) \# 3$ 年生存

sur_5_year $<-$ function $(\mathrm{x}) \operatorname{surv}(1 * 12 * 5, \mathrm{lp}=\mathrm{x}) \# 5$ 年生存

nom_sur <- nomogram(cox,fun=list(sur_3_year,sur_5_year),lp= F,funlabel=c('3-Year Survival','5-Year survival'),maxscale=100, fun.at=

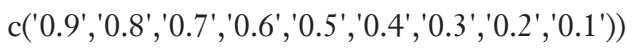

\# Painting nomogram

pdf("nom.pdf",15,10)

plot(nom_sur,xfrac $=0.25)$

dev.off()

f1 $<-\operatorname{coxph}($ formula $=$ Surv(survival_time, status $) ~$ Grade + PDW_PLT + ARHGAP9, data=tcga)

sum.surv<- summary(f1)

sum.surv

sum.surv\$concordance

C_index <- sum.surv\$concordance['C']

C_index

se $\quad<-$ sum.surv\$concordance['se(C)']

se

Upper95 <- (C_index $\left.+1.96^{*} \mathrm{se} \quad\right)$

Low95 <- (C_index $\left.-1.96^{*} \mathrm{se} \quad\right)$

\#\#95\% CI C-index

cbind( C_index, Low95, Upper95)

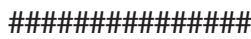

